\title{
Some Double Sequence Spaces of Fuzzy Real Numbers of Paranormed Type
}

\author{
Bipul Sarma \\ Department of Mathematics, Madhab Choudhury College-Gauhati University, Barpeta, Assam 781301, India \\ Correspondence should be addressed to Bipul Sarma; drbsar@yahoo.co.in
}

Received 7 November 2012; Revised 3 January 2013; Accepted 16 January 2013

Academic Editor: Pierpaolo D’Urso

Copyright (C) 2013 Bipul Sarma. This is an open access article distributed under the Creative Commons Attribution License, which permits unrestricted use, distribution, and reproduction in any medium, provided the original work is properly cited.

We study different properties of convergent, null, and bounded double sequence spaces of fuzzy real numbers like completeness, solidness, sequence algebra, symmetricity, convergence-free, and so forth. We prove some inclusion results too.

\section{Introduction}

Throughout the paper, a double sequence is denoted by $\left\langle X_{n k}\right\rangle$, a double infinite array of elements $X_{n k}$, where each $X_{n k}$ is a fuzzy real number.

The initial work on double sequences is found in Bromwich [1]. Later on, it was studied by Hardy [2], Móricz [3], Tripathy [4], Basarir and Sonalcan [5], and many others. Hardy [2] introduced the notion of regular convergence for double sequences.

The concept of paranormed sequences was studied by Nakano [6] and Simons [7] at the initial stage. Later on, it was studied by many others.

After the introduction of fuzzy real numbers, different classes of sequences of fuzzy real numbers were introduced and studied by Tripathy and Nanda [8], Choudhary and Tripathy [9], Tripathy et al. [10-13], Tripathy and Dutta [1416], Tripathy and Borgogain [17], Tripathy and Das [18], and many others.

Let $D$ denote the set of all closed and bounded intervals $X=\left[a_{1}, a_{2}\right]$ on $R$, the real line. For $X, Y \in D$, we define

$$
d(X, Y)=\max \left(\left|a_{1}-b_{1}\right|,\left|a_{2}-b_{2}\right|\right),
$$

where $X=\left[a_{1}, a_{2}\right]$ and $Y=\left[b_{1}, b_{2}\right]$. It is known that $(D, d)$ is a complete metric space.

A fuzzy real number $X$ is a fuzzy set on $R$, that is, a mapping $X: R \rightarrow I(=[0,1])$ associating each real number $t$ with its grade of membership $X(t)$.
The $\alpha$-level set $[X]^{\alpha}$ of the fuzzy real number $X$, for $0<$ $\alpha \leq 1$, is defined as $[X]^{\alpha}=\{t \in R: X(t) \geq \alpha\}$.

The set of all upper semicontinuous, normal, and convex fuzzy real numbers is denoted by $R(I)$, and throughout the paper, by a fuzzy real number, we mean that the number belongs to $R(I)$.

Let $X, Y \in R(I)$, and let the $\alpha$-level sets be $[X]_{\alpha}=$ $\left[a_{1}^{\alpha}, a_{2}^{\alpha}\right],[Y]_{\alpha}=\left[b_{1}^{\alpha}, b_{2}^{\alpha}\right]$, and $\alpha \in[0,1]$; the product of $X$ and $Y$ is defined by

$$
[X \otimes Y]_{\alpha}=\left[\min _{i, j \in\{1,2\}} a_{i}^{\alpha} \cdot b_{j}^{\alpha}, \max _{i, j \in\{1,2\}} a_{i}^{\alpha} \cdot b_{j}^{\alpha}\right] .
$$

\section{Definitions and Preliminaries}

A fuzzy real number $X$ is called convex if $X(t) \geq X(s) \wedge X(r)=$ $\min (X(s), X(t))$, where $s<t<r$.

If there exists $t_{0} \in R$ such that $X\left(t_{0}\right)=1$, then the fuzzy real number $X$ is called normal.

A fuzzy real number $X$ is said to be upper semicontinuous if, for each $\varepsilon>0, X^{-1}([0, a+\varepsilon))$, for all $a \in I$, is open in the usual topology of $R$.

The set $R$ of all real numbers can be embedded in $R(I)$. For $r \in R, \bar{r} \in R(I)$ is defined by

$$
\bar{r}(t)= \begin{cases}1, & \text { for } t=r \\ 0, & \text { for } t \neq r\end{cases}
$$



[19])

The absolute value, $|X|$ of $X \in R(I)$, is defined by (see, e.g.,

$$
\begin{gathered}
|X|(t)=\max \{X(t), X(-t)\} \quad \text { if } t \geq 0, \\
|X|(t)=0 \quad \text { if } t<0 .
\end{gathered}
$$

A fuzzy real number $X$ is called nonnegative if $X(t)=0$, for all $t<0$. The set of all nonnegative fuzzy real numbers is denoted by $R^{*}(I)$.

Let $\bar{d}: R(I) \times R(I) \rightarrow R$ be defined by

$$
\bar{d}(X, Y)=\sup _{0 \leq \alpha \leq 1} d\left([X]^{\alpha},[Y]^{\alpha}\right) .
$$

Then $\bar{d}$ defines a metric on $R(I)$.

The additive identity and multiplicative identity in $R(I)$ are denoted by $\overline{0}$ and $\overline{1}$, respectively.

A sequence $\left(X_{k}\right)$ of fuzzy real numbers is said to be convergent to the fuzzy real number $L$ if, for every $\varepsilon>0$, there exists $n_{0} \in N$ such that $\bar{d}\left(X_{k}, L\right)<\varepsilon$, for all $k \geq n_{0}$.

A sequence of fuzzy numbers $\left(f_{n}\right)$ converges to a fuzzy number $f$ if both $\lim _{n \rightarrow \infty}\left[f_{n}\right]_{\alpha}^{-}=[f]_{\alpha}^{-}$and $\lim _{n \rightarrow \infty}\left[f_{n}\right]_{\alpha}^{+}=$ $[f]_{\alpha}^{+}$hold for every $\alpha \in(0,1][20]$.

A sequence $\left(x_{n}\right)$ of generalized fuzzy numbers converges weakly to a generalized fuzzy number $x$ (and we write $x_{n} \stackrel{w}{\rightarrow}$ $x$ ) if distribution functions $\left(x_{n}^{l}\right)$ converge weakly to $x^{l}$ and $\left(x_{n}^{r}\right)$ converge weakly to $x^{r}[21]$.

A double sequence $\left(X_{n k}\right)$ of fuzzy real numbers is said to be convergent in Pringsheim's sense to the fuzzy real number $L$ if, for every $\varepsilon>0$, there exists $n_{0}, k_{0} \in N$ such that $\bar{d}\left(X_{n k}, L\right)<\varepsilon$, for all $n \geq n_{0}, k \geq k_{0}$.

A double sequence $\left(X_{n k}\right)$ of fuzzy real numbers is said to be regularly convergent if it converges in Pringsheim's sense, and the following limits exist:

$$
\begin{array}{ll}
\lim _{n} \bar{d}\left(X_{n k}, L_{k}\right)=0, & \text { for some } L_{k} \in R(I), \\
& \text { for each } k \in N, \\
\lim _{k} \bar{d}\left(X_{n k}, J_{n}\right)=0, & \text { for some } J_{n} \in R(I), \\
& \text { for each } n \in N .
\end{array}
$$

A fuzzy real number sequence $\left(X_{k}\right)$ is said to be bounded if $\sup _{k}\left|X_{k}\right| \leq \mu$, for some $\mu \in R^{*}(I)$.

For $r \in R$ and $X \in R(I)$, we define

$$
\begin{gathered}
r X(t)=X\left(r^{-1} t\right) \quad \text { if } r \neq 0, \\
r X(t)=\overline{0} \quad \text { if } r=0 .
\end{gathered}
$$

Throughout the paper ${ }_{2} w_{F},\left({ }_{2} \ell_{\infty}\right){ }_{F},{ }_{2} c_{F},\left({ }_{2} c_{0}\right){ }_{F},{ }_{2} c_{F}^{R}$, and $\left({ }_{2} c_{0}^{R}\right)_{F}$ denote the classes of all, bounded, convergent in Pringsheim's sense, null in Pringsheim's sense, regularly convergent, and regularly null fuzzy real number sequences, respectively.

A double sequence space $E_{F}$ is said to be solid (or normal) if $\left\langle Y_{n k}\right\rangle \in E_{F}$, whenever $\left|Y_{n k}\right| \leq\left|X_{n k}\right|$, for all $n, k \in N$, for some $\left\langle X_{n k}\right\rangle \in E_{F}$.
Let $K=\left\{\left(n_{i}, k_{i}\right): i \in N ; n_{1}<n_{2}<n_{3}<\cdots\right.$ and $k_{1}<$ $\left.k_{2}<k_{3}<\cdots\right\} \subseteq N \times N$, and let $E_{F}$ be a double sequence space. A $K$-step space of $E_{F}$ is a sequence space

$$
\lambda_{K}^{E}=\left\{\left\langle X_{n_{i} k_{i}}\right\rangle \in{ }_{2} w_{F}:\left\langle X_{n k}\right\rangle \in E_{F}\right\} .
$$

A canonical preimage of a sequence $\left\langle X_{n_{i} k_{i}}\right\rangle \in E_{F}$ is a sequence $\left\langle Y_{n k}\right\rangle$ defined as follows:

$$
Y_{n k}= \begin{cases}X_{n k} & \text { if }(n, k) \in K, \\ 0, & \text { otherwise. }\end{cases}
$$

A canonical preimage of a step space $\lambda_{K}^{E}$ is a set of canonical preimages of all elements in $\lambda_{K}^{E}$.

A double sequence space $E_{F}$ is said to be monotone if $E_{F}$ contains the canonical preimage of all its step spaces.

From the above definitions, we have the following remark.

Remark 1. A sequence space $E_{F}$ is solid $\Rightarrow E_{F}$ is monotone.

A double sequence space $E_{F}$ is said to be symmetric if $\left(X_{\pi(n, k)}\right) \in E_{F}$, whenever $\left(X_{n k}\right) \in E_{F}$, where $\pi$ is a permutation of $N \times N$.

A double sequence space $E_{F}$ is said to be sequence algebra if $\left(X_{n k} \otimes Y_{n k}\right) \in E_{F}$, whenever $\left(X_{n k}\right),\left(Y_{n k}\right) \in E_{F}$.

A double sequence space $E_{F}$ is said to be convergence-free if $\left(Y_{n k}\right) \in E_{F}$, whenever $\left(X_{n k}\right) \in E_{F}$, and $X_{n k}=\overline{0}$ implies that $Y_{n k}=\overline{0}$.

Sequences of fuzzy real numbers relative to the paranormed sequence spaces were studied by Choudhary and Tripathy [9].

In this paper, we introduce the following sequence spaces of fuzzy real numbers.

Let $p=\left\langle p_{n k}\right\rangle$ be a sequence of positive real numbers

$$
\left({ }_{2} \ell_{\infty}\right)_{F}(p)=\left\{\left\langle X_{n k}\right\rangle \in{ }_{2} w_{F}:\right.
$$

$$
\left.\sup _{n, k}\left\{\bar{d}\left(X_{n k}, \overline{0}\right)\right\}^{p_{n k}}<\infty\right\},
$$

$$
\begin{aligned}
{ }_{2} \mathcal{C}_{F}(p)=\left\{\left\langle X_{n k}\right\rangle \in{ }_{2} w_{F}: \lim _{n, k}\left\{\bar{d}\left(X_{n k}, L\right)\right\}^{p_{n k}}=0,\right. \\
\text { for some } L \in R(I)\} .
\end{aligned}
$$

For $L=\overline{0}$, we get the class $\left({ }_{2} c_{F}\right)_{0}(p)$.

Also a fuzzy sequence $\left\langle X_{n k}\right\rangle \in{ }_{2} c_{F}^{R}(p)$ if $\left\langle X_{n k}\right\rangle \in{ }_{2} c_{F}(p)$, and the following limits exist:

$$
\begin{array}{ll}
\lim _{n}\left\{\bar{d}\left(X_{n k}, L_{k}\right)\right\}^{p_{n k}}=0, & \text { for some } L_{k} \in R(I), \\
& \text { for each } k \in N, \\
\lim _{k}\left\{\bar{d}\left(X_{n k}, J_{n}\right)\right\}^{p_{n k}}=0, & \text { for some } J_{n} \in R(I), \\
& \text { for each } n \in N .
\end{array}
$$

For the class of sequences $\left({ }_{2} c_{F}^{R}\right)_{0}(p), L=L_{k}=J_{n}=0$.

We define $m_{F}(p)={ }_{2} c_{F}^{R}(p) \cap\left({ }_{2} \ell_{\infty}\right)_{F}(p),\left(m_{0}\right)_{F}(p)=$ $\left({ }_{2} c_{F}^{R}\right)_{0}(p) \cap\left({ }_{2} \ell_{\infty}\right)_{F}(p)$. 


\section{Main Results}

Theorem 2. Let $\left\langle p_{n k}\right\rangle$ be bounded. Then, the classes of sequences $\left({ }_{2} \ell_{\infty}\right)_{F}(p),{ }_{2} c_{F}^{R}(p),\left({ }_{2} c_{F}^{R}\right)_{0}(p), m_{F}(p)$, and $\left(m_{0}\right)_{F}(p)$ are complete metric spaces with respect to the metric defined by

$$
\begin{array}{r}
f(X, Y)=\sup _{n, k}\left\{\bar{d}\left(X_{n k}, Y_{n k}\right)\right\}^{p_{n k} / H}, \\
\text { where } H=\max \left(1, \sup p_{n k}\right) .
\end{array}
$$

Proof. We prove the result for ${ }_{2} \ell_{\infty}(p)$. Let $\left\langle X_{n k}^{i}\right\rangle$ be a Cauchy sequence in $\left({ }_{2} \ell_{\infty}\right)_{F}(p)$. Then, for a given $\varepsilon>0$, there exists $m_{0} \in N$ such that

$$
\begin{aligned}
& f\left(X_{n k}^{i}, X_{n k}^{j}\right)<\varepsilon<\varepsilon^{p_{n k} / H}, \quad \forall i, j \geq m_{0}, \\
& \Longrightarrow \bar{d}\left(X_{n k}^{i}, X_{n k}^{j}\right)<\varepsilon, \quad \forall i, j \geq m_{0} \\
& \Longrightarrow\left\langle X_{n k}^{j}\right\rangle_{j=1}^{\infty} \text { is a Cauchy sequence of fuzzy } \\
& \text { real number for each } n, k \in N .
\end{aligned}
$$

Since $R(I)$ is complete, there exist fuzzy numbers $X_{n k}$ such that $\lim _{j \rightarrow \infty} X_{n k}^{j}=X_{n k}$, for each $n, k \in N$.

Taking $j \rightarrow \infty$ in (13), we have

$$
f\left(X_{n k}^{i}, X_{n k}\right)<\varepsilon .
$$

Using the triangular inequality

$$
f\left(\left\langle X_{n k}\right\rangle, \overline{0}\right) \leq f\left(\left\langle X_{n k}\right\rangle,\left\langle X_{n k}^{j}\right\rangle\right)+f\left(\left\langle X_{n k}^{j}\right\rangle, \overline{0}\right),
$$

we have $\left\langle X_{n k}\right\rangle \in\left({ }_{2} \ell_{\infty}\right)_{F}(p)$. Hence, $\left({ }_{2} \ell_{\infty}\right)_{F}(p)$ is complete.

Property 1 . The space $\left({ }_{2} \ell_{\infty}\right)_{F}(p)$ is symmetric, but the spaces ${ }_{2} c_{F}(p),{ }_{2} c_{F}^{R}(p),\left({ }_{2} c_{F}\right)_{0}(p),\left({ }_{2} c_{F}^{R}\right)_{0}(p), m_{F}(p)$, and $\left(m_{0}\right)_{F}(p)$ are not symmetric.

Proof. Obviously the space $\left({ }_{2} \ell_{\infty}\right)_{F}(p)$ is symmetric. For the other spaces, consider the following example.

Example 3. Consider the sequence space ${ }_{2} c_{F}(p)$. Let $p_{1 k}=2$, for all $k \in N$ and $p_{n k}=3$, otherwise. Let the sequence $\left\langle X_{n k}\right\rangle$ be defined by

$$
X_{1 k}=\overline{1} \quad \forall k \in N
$$

and for $n>1$,

$$
X_{n k}(t)= \begin{cases}t+2, & \text { for }-2 \leq t \leq-1 \\ -t, & \text { for }-1 \leq t \leq 0 \\ 0, & \text { otherwise }\end{cases}
$$

Let $\left\langle Y_{n k}\right\rangle$ be a rearrangement of $\left\langle X_{n k}\right\rangle$ defined by

$$
Y_{n n}=\overline{1},
$$

and for $n \neq k$,

$$
Y_{n k}(t)= \begin{cases}t+2, & \text { for }-2 \leq t \leq-1 \\ -t, & \text { for }-1 \leq t \leq 0 \\ 0, & \text { otherwise. }\end{cases}
$$

Then, $\left\langle X_{n k}\right\rangle \in{ }_{2} c_{F}(p)$, but $\left\langle Y_{n k}\right\rangle \notin{ }_{2} c_{F}(p)$. Hence, ${ }_{2} c_{F}(p)$ is not symmetric. Similarly, it can be established that the other spaces are also not symmetric.

Theorem 4. The spaces $\left({ }_{2} \ell_{\infty}\right)_{F}(p),\left({ }_{2} c_{0}\right)_{F}(p),\left({ }_{2} c_{0}^{R}\right)_{F}(p)$, and $\left(m_{0}\right)_{F}(p)$ are solid.

Proof. Consider the sequence space $\left({ }_{2} \ell_{\infty}\right)_{F}(p)$. Let $\left\langle X_{n k}\right\rangle \in$ $\left({ }_{2} \ell_{\infty}\right)_{F}(p)$, and let $\left\langle Y_{n k}\right\rangle$ be such that $\bar{d}\left(Y_{n k}, \overline{0}\right) \leq \bar{d}\left(X_{n k}, \overline{0}\right)$.

The result follows from the inequality

$$
\left\{\bar{d}\left(Y_{n k}, \overline{0}\right)\right\}^{p_{n k}} \leq\left\{\bar{d}\left(X_{n k}, \overline{0}\right)\right\}^{p_{n k}} .
$$

Hence, the space $\left({ }_{2} \ell_{\infty}\right)_{F}(p)$ is solid. Similarly, the other spaces are also solid.

Property 2. The spaces ${ }_{2} c_{F}(p),\left({ }_{2} c^{R}\right)_{F}(p)$, and $m_{F}(p)$ are not monotone and hence are not solid.

Proof. The result follows from the following example.

Example 5. Consider the sequence space ${ }_{2} c_{F}(p)$. Let $p_{n k}=3$ for $n+k$ even and $p_{n k}=2$, otherwise. Let $J=\{(n, k): n+$ $k$ is even $\} \subseteq N \times N$. Let $\left\langle X_{n k}\right\rangle$ be defined by the following: for all $n, k \in N$,

$X_{n k}(t)$

$$
= \begin{cases}t+3, & \text { for }-3 \leq t \leq-2, \\ n t(3 n-1)^{-1}+3 n(3 n-1)^{-1}, & \text { for }-2 \leq t \leq-1+n^{-1}, \\ 0, & \text { otherwise }\end{cases}
$$

Then, $\left\langle X_{n k}\right\rangle \in{ }_{2} \mathcal{c}_{F}(p)$. Let $\left\langle Y_{n k}\right\rangle$ be the canonical preimage of $\left\langle X_{n k}\right\rangle_{J}$ for the subsequence $J$ of $N$. Then,

$$
Y_{n k}= \begin{cases}X_{n k}, & \text { for }(n, k) \in J, \\ \overline{0}, & \text { otherwise. }\end{cases}
$$

Then, $\left\langle Y_{n k}\right\rangle \notin{ }_{2} c_{F}(p)$. Thus, ${ }_{2} c_{F}(p)$ is not monotone. Similarly, the other spaces are also not monotone. Hence, the spaces $\left.{ }_{2} c_{F}(p),{ }_{2} c^{R}\right){ }_{F}(p)$, and $m_{F}(p)$ are not solid.

Property 3. The spaces $\left({ }_{2} \ell_{\infty}\right)_{F}(p),{ }_{2} c_{F}(p), \quad\left({ }_{2} c_{0}\right)_{F}(p)$, $\left({ }_{2} c^{R}\right)_{F}(p),\left({ }_{2} c_{0}^{R}\right)_{F}(p), m_{F}(p)$, and $\left(m_{0}\right)_{F}(p)$ are not convergence-free.

The result follows from the following example.

Example 6. Consider the sequence space ${ }_{2} c_{F}(p)$. Let $p_{1 k}=1$, for all $k \in N, p_{n k}=3$, otherwise. Consider the sequence $\left\langle X_{n k}\right\rangle$ defined by

$$
X_{1 k}=\overline{0}
$$


and for other values,

$$
X_{n k}(t)= \begin{cases}t+2, & \text { for }-2 \leq t \leq-1 \\ -n t(n+1)^{-1}+(n+1)^{-1}, & \text { for }-1 \leq t \leq n^{-1} \\ 0, & \text { otherwise }\end{cases}
$$

Let the sequence $\left\langle Y_{n k}\right\rangle$ be defined by

$$
Y_{1 k}=\overline{0},
$$

and for other values,

$$
Y_{n k}(t)= \begin{cases}1, & \text { for } 0 \leq t \leq 1 \\ (n-1)(n-1)^{-1}, & \text { for } 1 \leq t \leq n \\ 0, & \text { otherwise. }\end{cases}
$$

Then, $\left\langle X_{n k}\right\rangle \in{ }_{2} c_{F}(p)$, but $\left\langle Y_{n k}\right\rangle \notin{ }_{2} c_{F}(p)$. Hence, the space ${ }_{2} c_{F}(p)$ is not convergence-free. Similarly, the other spaces are also not convergence-free.

Theorem 7. $Z(p) \subseteq\left({ }_{2} \ell_{\infty}\right)_{F}(p)$, for $Z=\left({ }_{2} c^{R}\right)_{F},\left({ }_{2} c_{0}^{R}\right)_{F}, m_{F}$, $\left(m_{0}\right)_{F}$. The inclusions are strict.

Proof. Since convergent sequences are bounded, the proof is clear.

Theorem 8. Let $0<q_{i j} \leq p_{i j}<\infty$, for all $i, j \in N$. Then, $Z(p) \subseteq Z(q)$ for $Z={ }_{2} c_{F},\left({ }_{2} c^{R}\right)_{F},\left({ }_{2} c_{F}\right)_{0},\left({ }_{2} c_{0}^{R}\right)_{F}, m_{F},\left(m_{0}\right)_{F}$.

Proof. Consider the sequence spaces ${ }_{2} c_{F}(p)$ and ${ }_{2} c_{F}(q)$. Let $\left\langle X_{n k}\right\rangle \in{ }_{2} c_{F}(p)$.

Then, $\left\{\bar{d}\left(X_{n k}, L\right)\right\}^{p_{n k}}<\varepsilon$, for all $n \geq n_{0}, k \geq k_{0}$.

The result follows from the inequality $\left\{\bar{d}\left(X_{n k}, L\right)\right\}^{q_{n k}} \leq$ $\left\{\bar{d}\left(X_{n k}, L\right)\right\}^{p_{n k}}$.

Theorem 9. The spaces $\left({ }_{2} \ell_{\infty}\right)_{F}(p),{ }_{2} c_{F}(p), \quad\left({ }_{2} c_{0}\right)_{F}(p)$, $\left({ }_{2} c^{R}\right)_{F}(p),\left({ }_{2} c_{0}^{R}\right)_{F}(p), m_{F}(p)$, and $\left(m_{0}\right)_{F}(p)$ are sequence algebras.

Proof. Consider the sequence space $\left({ }_{2} c_{0}\right)_{F}(p)$. Let $\left\langle X_{n k}\right\rangle$, $\left\langle Y_{n k}\right\rangle \in\left({ }_{2} c_{0}\right)_{F}(p)$. Then, the result follows immediately from the inequality

$$
\left\{\bar{d}\left(X_{n k} Y_{n k}, \overline{0}\right)\right\}^{p_{n k}} \leq\left\{\bar{d}\left(X_{n k}, \overline{0}\right)\right\}^{p_{n k}}\left\{\bar{d}\left(Y_{n k}, \overline{0}\right)\right\}^{p_{n k}} .
$$

\section{Acknowledgment}

The author's work is supported by UGC Project no. F. 5294/2009-10 (MRP/NERO).

\section{References}

[1] T. J. I. A. Bromwich, An Introduction to the Theory of Infinite Series, Macmillan, New York, NY, USA, 1965.

[2] G. H. Hardy, "On the convergence of certain multiple series," Mathematical Proceedings of the Cambridge Philosophical Society, vol. 19, pp. 86-95, 1917.
[3] F. Móricz, "Extensions of the spaces $c$ and $c_{0}$ from single to double sequences," Acta Mathematica Hungarica, vol. 57, no. 1-2, pp. 129-136, 1991.

[4] B. C. Tripathy, "Statistically convergent double sequences," Tamkang Journal of Mathematics, vol. 34, no. 3, pp. 231-237, 2003.

[5] M. Basarir and O. Sonalcan, "On some double sequence spaces," Journal of Indian Academy of Mathematics, vol. 21, no. 2, pp. 193200, 1999.

[6] H. Nakano, "Modulared sequence spaces," Proceedings of the Japan Academy, vol. 27, pp. 508-512, 1951.

[7] S. Simons, "The sequence spaces $l\left(p_{v}\right)$ and $m\left(p_{v}\right)$," Proceedings of the London Mathematical Society 3, vol. 15, no. 1, pp. 422-436, 1965.

[8] B. K. Tripathy and S. Nanda, "Absolute value of fuzzy real numbers and fuzzy sequence spaces," Journal of Fuzzy Mathematics, vol. 8, no. 4, pp. 883-892, 2000.

[9] B. Choudhary and B. C. Tripathy, "On fuzzy real-valued $\ell(p)^{F}$ sequence spaces," in Proceedings of the International 8th Joint Conference on Information Sciences (10th International Conference on Fuzzy Theory and Technology), pp. 184-190, Salt Lake City, Utah, USA, July 2005.

[10] B. C. Tripathy and A. Baruah, "New type of difference sequence spaces of fuzzy real numbers," Mathematical Modelling and Analysis, vol. 14, no. 3, pp. 391-397, 2009.

[11] B. C. Tripathy and A. Baruah, "Nörlund and Riesz mean of sequences of fuzzy real numbers," Applied Mathematics Letters, vol. 23, no. 5, pp. 651-655, 2010.

[12] B. C. Tripathy and A. Baruah, "Lacunary statically convergent and lacunary strongly convergent generalized difference sequences of fuzzy real numbers," Kyungpook Mathematical Journal, vol. 50, no. 4, pp. 565-574, 2010.

[13] B. C. Tripathy, A. Baruah, M. Et, and M. Gungor, "On almost statistical convergence of new type of generalized difference sequence of fuzzy numbers," Iranian Journal of Science and Technology A, vol. 36, no. 2, pp. 147-155, 2012.

[14] B. C. Tripathy and A. J. Dutta, "On fuzzy real-valued double sequence space ${ }_{2} l_{F}^{p}$, Mathematical and Computer Modelling, vol. 46, no. 9-10, pp. 1294-1299, 2007.

[15] B. C. Tripathy and A. J. Dutta, "Bounded variation double sequence space of fuzzy real numbers," Computers \& Mathematics with Applications, vol. 59, no. 2, pp. 1031-1037, 2010.

[16] B. C. Tripathy and A. J. Dutta, "On I-acceleration convergence of sequences of fuzzy real numbers," Journal Mathematical Modelling and Analysis, vol. 17, no. 4, pp. 549-557, 2012.

[17] B. C. Tripathy and S. Borgogain, "Some classes of difference sequence spaces of fuzzy real numbers defined by Orlicz function," Advances in Fuzzy Systems, vol. 2011, Article ID 216414, 6 pages, 2011.

[18] B. C. Tripathy and P. C. Das, "On convergence of series of fuzzy real numbers," Kuwait Journal of Science \& Engineering, vol. 39, no. 1, pp. 57-70, 2012.

[19] O. Kaleva and S. Seikkala, "On fuzzy metric spaces," Fuzzy Sets and Systems, vol. 12, no. 3, pp. 215-229, 1984.

[20] J. Hančl, L. Mišík, and J. T. Tóth, "Cluster points of sequences of fuzzy real numbers," Soft Computing, vol. 14, no. 4, pp. 399-404, 2010.

[21] D. Zhang, "A natural topology for fuzzy numbers," Journal of Mathematical Analysis and Applications, vol. 264, no. 2, pp. 344353, 2001. 


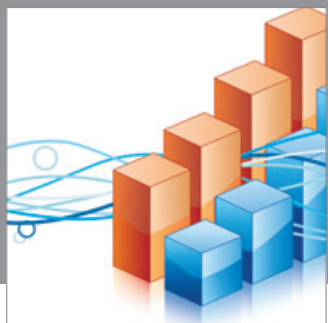

Advances in

Operations Research

mansans

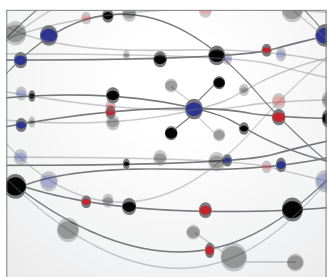

The Scientific World Journal
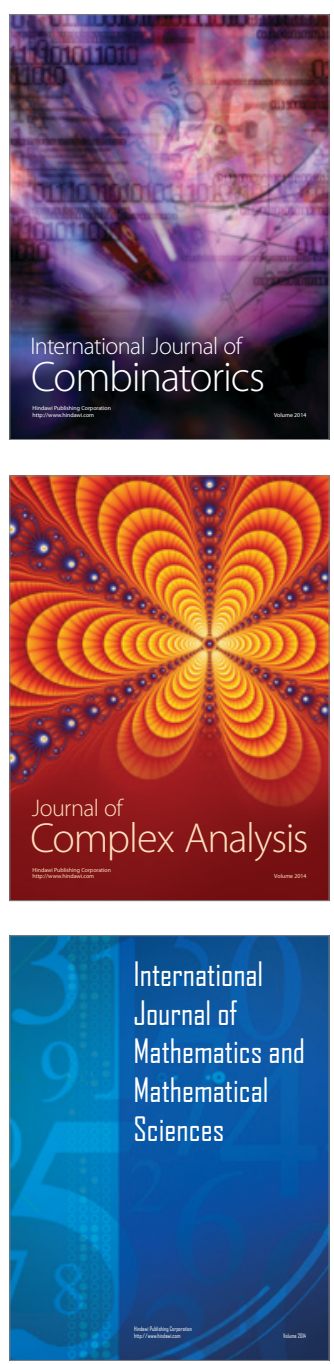
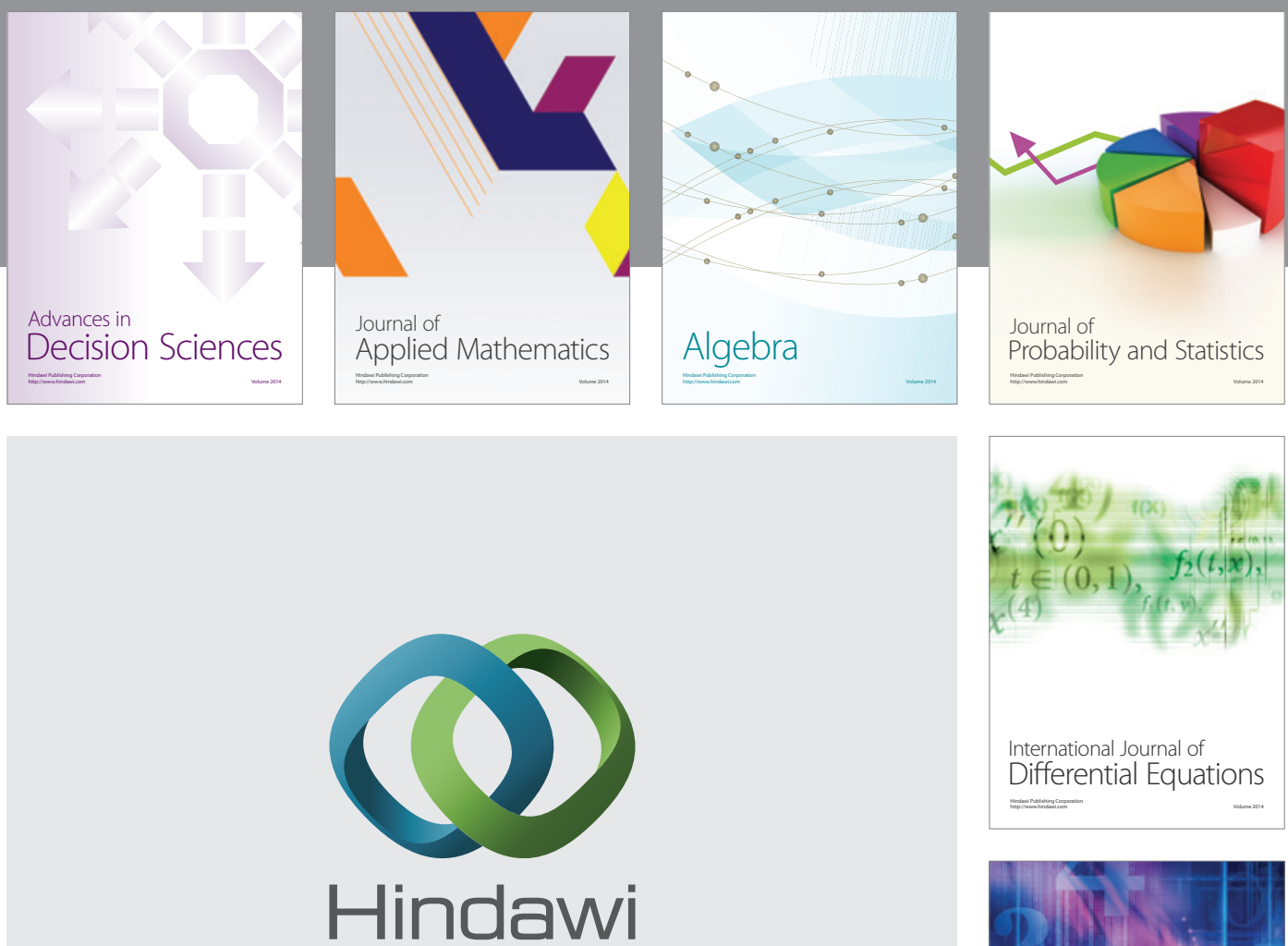

Submit your manuscripts at http://www.hindawi.com
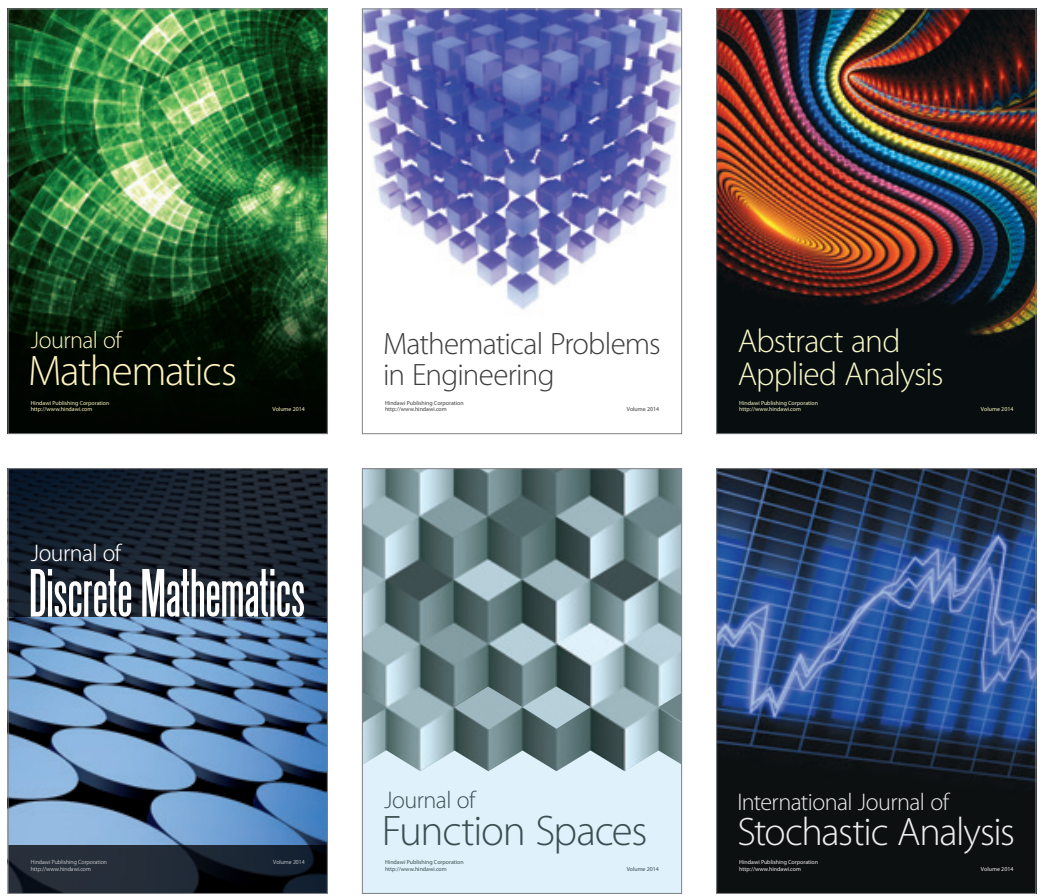

Journal of

Function Spaces

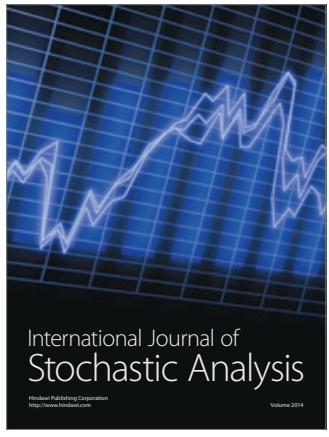

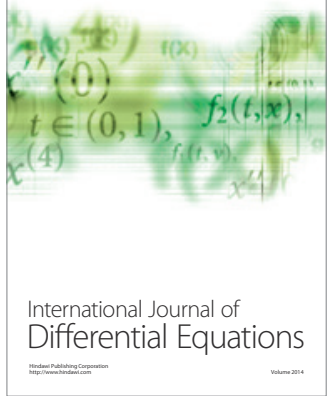
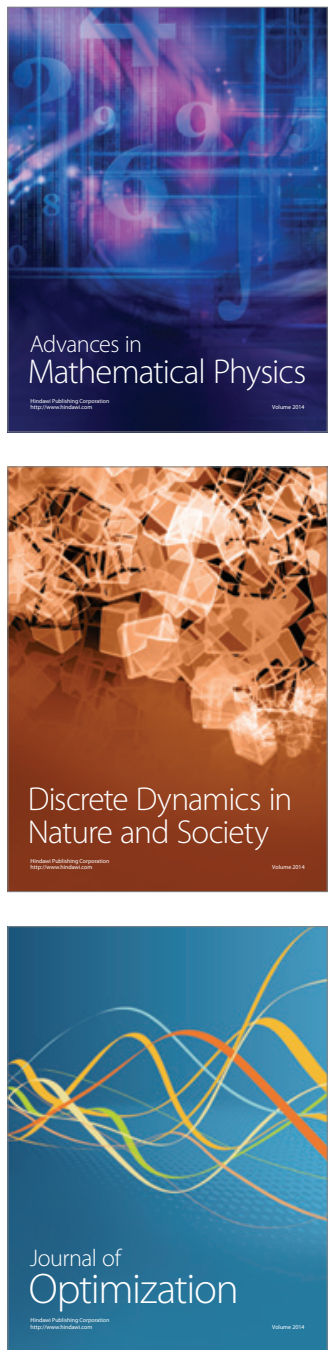